Davidson, A. I., Halstead, S. K., Goodfellow, J. A., Chavada, G., Mallik, A., Overell, J., Lunn, M. P., McConnachie, A., van Doorn, P. and Willison, H. J. (2017) Inhibition of complement in Guillain-Barré Syndrome: the ICA-GBS study. Journal of the Peripheral Nervous System, 6, pp. 4-12. (doi:10.1111/jns.12194)

There may be differences between this version and the published version. You are advised to consult the publisher's version if you wish to cite from it.

This is the peer-reviewed version of the following article: Davidson, A. I., Halstead, S. K., Goodfellow, J. A., Chavada, G., Mallik, A., Overell, J., Lunn, M. P., McConnachie, A., van Doorn, P. and Willison, H. J. (2017) Inhibition of complement in Guillain-Barré Syndrome: the ICAGBS study. Journal of the Peripheral Nervous System, 6, pp. 4-12, which has been published in final form at 10.1111/jns.12194. This article may be used for non-commercial purposes in accordance with Wiley Terms and Conditions for Self-Archiving.

\title{
http://eprints.gla.ac.uk/131370/
}

Deposited on: 19 December 2016 


\section{Inhibition of Complement in Guillain-Barré Syndrome: The ICA-GBS}

\section{Study}

Amy I Davidson ${ }^{1,2}$, Susan K Halstead ${ }^{1}$, John A Goodfellow ${ }^{1,2}$, Govind Chavada ${ }^{2}$, Arup Mallik $^{3}$, James Overell ${ }^{2}$, Michael P Lunn ${ }^{4}$, Alex McConnachie ${ }^{5}$, Pieter van Doorn ${ }^{6}$, Hugh J Willison ${ }^{1,2}$.

1. Institute of Infection, Immunity and Inflammation, College of Medical, Veterinary and Life Sciences, University of Glasgow

2. Department of Neurology, Institute of Neurological Sciences, Southern General Hospital, 1345 Govan Road, Glasgow

3. Department of Clinical Neurophysiology, Institute of Neurological Sciences, Southern General Hospital, 1345 Govan Road, Glasgow

4. Department of Neurology, National Hospital for Neurology and Neurosurgery, Queen Square, London

${ }^{5}$ Robertson Centre for Biostatistics, Boyd Orr Building, University of Glasgow.

6. Department of Neurology, Erasmus MC, Rotterdam

Corresponding author:

Hugh J Willison

Glasgow Biomedical Research Centre, Room B330, 120 University Avenue, Glasgow G12 8TA, Scotland, UK.

Tel: 44141330 8287; Fax: 44141330 4600;

email: Hugh.Willison@glasgow.ac.uk

This article has been accepted for publication and undergone full peer review but has not been through the copyediting, typesetting, pagination and proofreading process, which may lead to differences between this version and the Version of Record. Please cite this article as doi: 10.1111/jns. 12194

This article is protected by copyright. All rights reserved. 


\section{Abstract}

The outcome of Guillain-Barré syndrome remains unchanged since plasma exchange and intravenous immunoglobulin were introduced over 20 years ago. Pathogenesis studies on GBS have identified the terminal component of complement cascade as a key disease mediator and therapeutic target. We report the first use of terminal complement pathway inhibition with eculizumab in humans with GBS. In a randomised, double-blind, placebo-controlled trial, 28 subjects eligible on the basis of GBS disability grade of at least 3 were screened, of whom $8(29 \%)$ were randomised. Five received eculizumab for four weeks, alongside standard intravenous immunoglobulin treatment. The safety outcomes, monitored via adverse events capture, showed eculizumab to be well tolerated and safe when administered in conjunction with IVIg. Primary and secondary efficacy outcomes in the form of GBS disability scores, MRC sum scores, Rasch Overall Disability Scores and Overall Neuropathy Limitation Scores are reported descriptively. For the primary efficacy outcome at 4 weeks after recruitment, 2 of 2 placebo and 2 of 5 eculizumab-treated subjects had improved by 1 or more grades on the GBS disability score. Although the small sample size precludes a statistically meaningful analysis, these pilot data indicate further studies on complement inhibition in GBS are warranted.

Key words: Guillain-Barré syndrome, complement, membrane attack complex, intravenous immunoglobulin, clinical trial, therapy, eculizumab. 


\section{Introduction}

Over 20 years after their introduction, intravenous immunoglobulin (IVIg) and plasma exchange remain the only proven therapies for Guillain-Barré syndrome (GBS) (Hughes et al., 2014). Since the use of these interventions became routine in clinical practice, no further immunomodulatory therapies have been shown to significantly ameliorate the clinical course of GBS, whether used alone or in conjunction with IVIg (Hughes et al., 2013).

IgG anti-ganglioside antibodies are strongly implicated in the pathogenesis of at least some forms of GBS (Willison 2005; Willison et al., 1999) by activating complement during the acute inflammatory phase. Human biopsies from GBS subjects reveal complement deposits in nerves (Griffin et al., 1996). Data from animal models has demonstrated that inhibition of the terminal components of the complement cascade can abrogate nerve damage (Goodyear et al., 1999; Greenshields et al., 2009; Halstead et al., 2008; O'Hanlon et al., 2003; van Schaik et al., 1995; Yuki et al., 2001) thus making complement an attractive therapeutic target.

Eculizumab (Soliris ${ }^{\mathrm{TM}}$ ) is currently the only commercially available complement C5 inhibitor approved for use in man. It is a humanised anti-C5 neutralising antibody which prevents the formation of MAC (Rother et al., 2007). In 2011, an open label clinical trial of eculizumab in multifocal motor neuropathy (MMN) provided valuable data on serum eculizumab concentrations required to inhibit MAC formation in subjects receiving concomitant IVIg (Fitzpatrick et al., 2011). We report here an exploratory study of eculizumab used as an add-on to IVIg in GBS. 


\section{Materials and Methods}

\section{Patients}

Patients that fulfilled the NINDS criteria for diagnosis of GBS (Asbury and Cornblath 1990) were included. Entry criteria required participants to be at least 18 years of age, within 2 weeks of GBS symptom onset, have a GBS disability score (GBS DS) of at least 3 (defined as inability to walk $10 \mathrm{~m}$ unaided), with no confounding medical conditions. Full inclusion and exclusion criteria are documented in Table 1. Regional ethical approval was given for the trial protocol and supporting documentation (REC13/SS/0117). All trial conduct was in keeping with the Declaration of Helsinki. The trial is registered on EudraCT database (unique no. 2013-000228-33) and Clinicaltrials.gov (NCT02029378). The trial was investigator-led, co-sponsored by the University of Glasgow and NHS Greater Glasgow and funded by Alexion Pharmaceuticals, Cheshire, Connecticut, USA.

\section{Study design}

Inhibition of Complement in Guillain Barré Syndrome (ICA-GBS) was designed as a phase 2, single centre, 2:1 randomised, double-blind, placebo-control trial aiming to recruit 30 patients over a 2 year period (August 2014 - July 2016) with severe GBS. Randomisation and concealed allocation were performed by computer and an interactive web response system. In both arms, participants received IVIg at $2 \mathrm{~g} / \mathrm{kg}$ $(0.4 \mathrm{~g} / \mathrm{kg} /$ day for 5 days). All participants were enrolled for a 6 month period, comprising 8 visits, at weeks $0,1,2,3,4,8,13$ and 26 . Active drug (eculizumab, 900mg) or 
placebo was given weekly (weeks 1, 2, 3 and 4) for the first 4 weeks. Concomitant antibiotics (Ciprofloxacin 400mg oral/500mg IV once weekly) were given to all participants for the first 10 weeks for meningococcal prophylaxis in the absence of an opportunity to vaccinate eculizumab recipients. When the first dose of study drug was administered, all participants were also receiving IVIg therapy (2g/kg in 5 divided doses over 5 days) at various timepoints. Outcome data, as denoted in Table 2, were collected throughout the trial and comprised GBS DS (Hughes et al., 1978) and MRC sum score (MRC SS) (Kleyweg et al., 1991) and both collected weekly. The R-ODS (van Nes et al., 2011) and ONLS (Draak et al., 2014) were collected at baseline, weeks 4, 8, 13 and 26 visits (Table 2). For full descriptions of scales and scores, see Appendix 3A-D.

\section{Study outcomes}

The primary safety endpoint was the incidence of adverse events (AE) and serious adverse events (SAE) after treatment with eculizumab and IVIg compared to placebo controls who received IVIg only. Safety was recorded via AE and SAE reporting occurring during the treatment period and for unresolved SAEs the subsequent 6 months. The primary efficacy endpoint was defined as improvement by one or more grades in GBS DS at 4 weeks. The secondary endpoints are documented in Table 2.

\section{Electrophysiology}

Routine clinical electrophysiological data were collected, with all subjects having a least one detailed study, recording from at least four nerve and muscle groups. These data 
were then analysed by one clinical neurophysiologist (AM) and compared to the Rajabally diagnostic criteria (Rajabally et al., 2015).

\section{Laboratory and Pharmacological monitoring}

Blood samples were taken prior to each eculizumab or placebo dose, and assays were performed at baseline for anti-ganglioside antibodies by enzyme-linked immunosorbent assay (ELISA) (Willison et al., 1999) and microarray (Cao-Lormeau et al., 2016).

\section{Statistics}

The study protocol specified that a statistical analysis plan (SAP) would be formulated prior to unblinding the trial. Since the study only recruited 8 participants of the intended 30 , it was agreed that there would be no value to estimate treatment effect difference, and the final SAP consisted of descriptive analyses only.

\section{Results}

\section{Subjects}

There were an unexpectedly large number of exclusions of potentially eligible subjects at pre-screening. Thus of the 28 subjects with severe GBS over the 2 year study period who underwent pre-screening, 20 subjects failed to enter the trial for a number of reasons (Figure 1, Appendix Table 1). Twelve of these subjects declined to participate, the most common reason being concern about the perceived risks of meningitis and infection associated with the trial drug. Eight were excluded for other reasons including 
concomitant pyrexial illness, past history of meningococcal CNS infection, and inability to comply with study schedules. Table 3 lists baseline clinical characteristics of recruited subjects.

Eight subjects were recruited and randomised. One randomised patient was withdrawn due to an exclusion criterion (incidental chest neoplasm identified on CT scanning) arising after consent was given, but before any baseline visit actions were undertaken. All 7 remaining subjects received standard IVIg treatment at $0.4 \mathrm{~g} / \mathrm{kg}$ per day for 5 days, with the first trial visit and dosing occurring during this time period. Of the 7 who received trial infusions and completed the study, 5 received eculizumab (subjects E1E5) and 2 received placebo (subjects $P 1-P 2$ ). One patient (E3), who was gradually improving from their GBS disability over the course of the study, died suddenly of septic shock at week 21 . All other subjects $(n=6)$ completed the predefined 26 week observation period and outcome measures.

All 7 subjects who completed the trial had clinical features that were typical for GBS with largely symmetrical motor weakness, reflex loss and elevated cerebrospinal fluid (CSF) protein. Five subjects had associated anti-ganglioside antibodies (Table 3).

Electrophysiological studies (Appendix 4) showed that amongst the eculizumab-treated subjects, $2(E 1, E 2)$ had AMAN/AMSAN pattern and 2 had AIDP patterns. One subject (E5) had equivocal studies. Both placebo patients had AIDP electrophysiological patterns. 


\section{Primary outcome - safety and tolerability}

Administration of the first dose of study drug occurred at least one hour post IVIg infusion. One patient reported a rash after the third dose of study drug; otherwise no infusion reactions were noted. Of the 5 who received eculizumab, 2 did not fully complete the 4 scheduled infusions. One patient (E3) received 2 out of 4 intended doses (missing weekly dose 3 and 4 ) and the other patient (E4) received 3 out of 4 (missing weekly dose 3). In both subjects, treatment was withheld because of active respiratory infection with pyrexia and sepsis as per protocol.

\section{Adverse Events}

Adverse events were captured for the duration of the trial (Table 4 and Appendix Table 2). For all 7 subjects who completed the full treatment phase of the trial, 29 AEs were reported. Twenty five were in the eculizumab arm and four in the placebo arm. The 2 most commonly reported AEs, occurring in either group, were derangements in liver function (rising liver transaminases) and infections. In the 5 reported cases of liver function derangement, 3 subjects had mildly deranged transaminases prior to infusion of trial medications. All liver function test derangements had resolved by the end of the trial visits. Of those with an infectious $\mathrm{AE}$, four had lower respiratory tract infections and one had acute cholecystitis.

Four SAEs occurred in the eculizumab cohort and none in the control cohort. Three were deemed as having no causality to the drug intervention and one with possible causality to the drug intervention. Subject E3 had a lower respiratory tract infection preventing administration of trial drug (possible causality), and died at week 21 due to 
septic shock and multi-organ failure (non-causal). Subject E4 had opioid toxicity requiring high dependency unit admission (non-causal) and resulting in an aspiration pneumonia leading to intensive care unit admission (non-causal).

\section{Primary efficacy endpoint}

By 4 weeks, 2 of 2 placebo and 2 of 5 eculizumab-treated subjects had improved by 1 or more grades on the GBS DS (Figure 2A, Table 4).

\section{Secondary Outcome Measurements}

\section{Time to improvement of one GBS DS point}

Of the 5 who received eculizumab, 3 (E1, E2, E5, 60\%) achieved an improvement of 1 GBS DS grade over the duration of the trial (Figure 2A). This was achieved by week 2 (E1), week $3(E 2)$, and week $13(E 5)$. The placebo-treated subjects achieved improvements of 1 GBS DS grade by week 1 (P1) and week 2 (P2) (Table 4, Figure 2). One of 5 eculizumab-treated subjects (E2) and 1 of the 2 placebo-treated subjects (P1) achieved a GBS DS score of 2 or less (independently walking) by week 8 of the trial. By week 13, 1 further eculizumab-treated patient (E1) and 1 further placebo-treated patient had also reached this outcome. Three of five eculizumab-treated subjects failed to achieve independent walking (GBS DS 2) by 6 months. One eculizumab-treated patient (E3) died of sepsis whilst still being ventilated at week 21. During the period prior to his death, motor function had been improving, as reflected in other scoring scales (E3 data from week 13 is displayed). 


\section{Rasch-built Overall Disability Score (R-ODS)}

At week 4, 2 of 5 (40\%) eculizumab-treated subjects (E1 and E2) had an increase of at least 6 points on the R-ODS (with an 11 point and 38 point improvement respectively), whilst one further patient (E5) had an improvement of 5 points (Figure 2B). Both of the remaining subjects were very severely affected. One remained static with a R-ODS score of 0 (E3) and one deteriorated by 11 points (falling from 11 to 0 ; E4). Both placebo-treated subjects had achieved at least 6 point improvement at week 4 , having improved by $41(E 1)$ and 15 (E2) points. At week 26, 4 of 5 eculizumab-treated subjects had improved by at least 6 points (range from 16 to 74 points), as had both placebotreated subjects, achieving a 94 point (P1) and 64 point (P2) improvement.

\section{MRC sum score (MRC SS)}

At week 4, 2 eculizumab-treated subjects (E3, E4) had regressed in their MRC SS

(Figure 2C). All other subjects ( 3 treated and 2 placebo) improved by at least one point. At week 26, 4 eculizumab-treated pateints had improved to near normal (range 54-59). At week 13, the MRC SS of E3 was 26. At 26 weeks, the 2 placebo-treated subjects had recovered (P1, 60; $P 2,58)$.

\section{Overall Neuropathy Limitation Score (ONLS)}

Of the 5 eculizumab-treated subjects, 2 subjects (E1 and E2) had an improvement of at least 1 point (3- and 8-point improvement respectively) at 4 weeks (Figure 2D). Four eculizumab-treated subjects had an improvement of at least 1 point by week 26 (range 
from 1 to 10 points). Patient E3 had improved by 1 point at week 13, and died at week 21. Both placebo subjects had improved by 1 point at week 4 , with an overall improvement of 7 (P1) and 10 (P2) ONLS points by trial completion.

\section{Ventilation requirements and Intensive Care Unit Admissions}

Three eculizumab-treated subjects required intensive care unit (ICU) admission, with 2 eculizumab-treated subjects (E3 and E4) requiring mechanical ventilation (Table 4). Subject E3 had a progressive bulbar palsy and was electively intubated for airway protection before receiving eculizumab doses, and subject E4 required mechanical ventilation due to pneumonia and sepsis during week 2 and 3 of their admission after starting eculizumab doses. Patient E3 was ventilator dependent throughout the trial. Patient 4 was ventilated for 10 days, though due to bulbar weakness retained a tracheostomy for 5 months. Patient E2 required high dependency care with high flow oxygen and non-invasive ventilation for 5 days, but did not require invasive mechanical ventilation. Neither placebo-treated subjects required ICU level care.

Two eculizumab-treated subjects required inotropic support for unstable blood pressure. Patient E3 had a combination of autonomic dysfunction secondary to GBS and sepsis, and required inotropic support for 6 weeks. Patient E4 had sepsis-related hypotension and required inotropic support for 4 days. Neither placebo-treated patient required cardiovascular support.

\section{Observed versus predicted outcome}


The mEGOS scale (Appendix 3E) was used to estimate predicted outcomes in all subjects. This was compared to observed outcome (Table 5). Whilst these comparative data are only probabilistic, subjects E4 and E5 appeared to underperform as manifested by the inability walk independently at week 26 despite an 85\% (E4) and 93\% (E5) probability of being able to do so, as defined by their mEGOS score. Excepting case E3 who died, other subjects conformed to their predicted probabilities by week 26 or earlier. The mEGRIS scale (Appendix 3F) was used to predict requirement of mechanical ventilation in the first week of illness. This was compared to observed outcome. One patient $(E 3)$ was predicted high risk (mEGRIS $=5$ ) and required ventilation at day 5 of his illness.

\section{Discussion}

This phase 2 study was designed to assess the safety and tolerability of eculizumab administration concomitantly with IVIg in subjects with severe GBS (GBS DS 3 or greater). These data show that the combination therapy was safe and well tolerated. The most common AEs reported were infection and deterioration in liver function, both of which are recognised complications of eculizumab administration but are also common complications of GBS (Oomes et al., 1996). Administering the full doses of eculizumab was prevented in 2 subjects due to sepsis, as outlined in the study protocol. This may pose a problem in severely affected subjects with GBS, as they are at a high risk for ventilator acquired pneumonia, aspiration due to bulbar weakness, and urinary tract sepsis due to indwelling urinary catheters. The effect of missed drug doses on attenuating systemic complement inhibition also needs to be considered, although this 
was not formally evaluated through serum complement activity measurement in this study. The infusions themselves were well tolerated, with only one reported rash. The concomitant ciprofloxacin was also well tolerated and meningitis was not seen. The single fatality, occurring in an eculizumab-treated patient, was deemed to be unrelated to the study drug as it occurred in week 21 , over 18 weeks after its last administration and well beyond its functional half-life.

Recruitment into the trial was unexpectedly difficult. Many participants declined, despite being eligible on other grounds. Overwhelmingly, the perceived risk and fear of meningitis, a known complication of eculizumab discussed during informed consent, was the critical element in participants declining. Whether the excluded subjects introduced a selection bias is not known as their outcomes are unknown; nevertheless this factor should be considered.

The small sample size means the efficacy results are not amenable to statistical analysis. No clear trends in efficacy were apparent but this is not unexpected in such a small sample size. Similarly, a small study is potentially vulnerable to differences in baseline characteristics that cannot be balanced in a controlled way. Larger studies would be required for such analysis. The overall course in these severely affected GBS subjects did not seem out of the ordinary, varying in range from full recovery to death. Neurophysiological studies and anti-ganglioside antibody analysis revealed a mixed cohort, with some showing markers typical for axonal GBS, and others not (Goodfellow and Willison 2016).

This study concludes that eculizumab appears well tolerated in acute GBS subjects and can be given safely in conjunction with IVIg. Although it is tempting to 
speculate on the varied clinical outcome following eculizumab in this group of patients the small sample size precludes a meaningful assessment of efficacy. Efficacy data from another ongoing Japanese phase 2 study of similar design (JET-GBS, ClinicalTrials.gov NCT02493725) are awaited with interest. The study also illustrates the challenges of testing novel immunotherapies in GBS subjects with sepsis. Difficulties in recruitment and ongoing drug administration were encountered, principally due to infection considerations related to both perceived risks of study drug and their frequent occurrence in GBS. These issues need to be carefully considered when designing future studies if a recruitment rate of under $30 \%$ of eligible subjects is estimated for phase 3 trials, study populations need to be at 3 to 4 times the recruitment target. Advances in treatment of GBS are urgently required, and universally adopted, adaptively designed trial protocols for assessing new pathway-specific immunotherapies that does not depend upon large randomized control trials would be advantageous for accelerating progress.

\section{Acknowledgements}

The authors would like to acknowledge the ICA-GBS independent data monitoring committee (DMC): Chairperson Prof D Cornblath (Dept of Neurology, Johns Hopkins University, Baltimore, USA), Prof Dr HP Hartung (Dept of Neurology, Heinrich-HeineUniversität Düsseldorf, Germany) Prof D Grossett (Dept of Neurology, Institute of Neurological Sciences, Glasgow, UK) and Dr S Rinaldi (Dept of Neurology, John Radcliffe Hospital, Oxford, UK). We thank the Clinical Research Facility in Glasgow, in 
particular Ms Lisa Jolly for assistance in project management, the clinical nursing team for their assistance in dosing and monitoring participants, and Elizabeth Douglas and colleagues in Trial Pharmacy, NHS Greater Glasgow and Clyde, for storage and supply of medicinal products. We thank data management, IT and statistical staff in the Robertson Centre for Biostatistics, University of Glasgow (S Kean, R Wilson, C Haig, B Francq, N Hillen, J McHugh).

Table 1. Inclusion and exclusion criteria

\section{Inclusion criteria}

1. Written informed consent, or witnessed verbal informed consent

2. Male or female $\geq 18$ years of age.

3. Participants diagnosed with GBS according to NINDS diagnostic criteria.

4. Onset of weakness due to GBS within 2 weeks of enrolment.

5. Patients who are being considered for or already on IVlg treatment.

6. Unable to walk 10 meters independently (grade $\geq 3$ on GBS disability scale).

7. First dose of eculizumab must be started within 2 weeks from onset of weakness and any time during the IVIg treatment period.

\section{Exclusion Criteria}

1. Pregnant, lactating women or participants who wish to become pregnant during the study period and for 5 months following treatment completion.

2. Participants who are being considered for or already on plasma exchange.

3. Patient shows clear clinical evidence of a polyneuropathy caused by e.g. diabetes mellitus (except mild sensory), alcoholism, severe vitamin deficiency, and porphyria.

4. Patient received immunosuppressive ${ }^{*}$ treatment during the last month.

5. Patient known to have a severe concurrent disease, inability to comply with study 
related procedures or appointments during 6 months.

6. Any condition that in the opinion of the investigator could increase the patient's risk by participating in the study or confound the outcome of the study.

7. Enrolment in another CTIMP 6 months prior to consent

8. Contraindications to the administration of eculizumab:

a) Unresolved $N$. Meningitidis infection or history of meningococcal infection.

b) Unsuitable for antibiotic prophylaxis, known hypersensitivity to eculizumab, murine proteins or to any of the excipients

c) Known or suspected hereditary complement deficiencies.

d) Women of child-bearing potential (WoCBP) who are unwilling to use effective contraception during the eculizumab treatment period and for a minimum of 5 months thereafter.

CTIMP, Clinical Trial of an Investigational Medicinal Product; NINDS, National Institute of Neurological Disorders and Stroke; IVIG, Intravenous immunoglobulin.

*including any dose of steroids greater than the equivalent of $20 \mathrm{mg}$ Prednisolone.

Table 2. Study outcomes

\section{Primary outcomes}

1. To determine the incidence of serious adverse event/adverse events after treatment with eculizumab and IVIg compared to placebo control

2. Improve one or more grade in functional outcome (GBS disability score) at 4 weeks

Secondary outcomes

1. Ability to walk unaided (GBS disability score $\leq 2$ ) at 8 weeks

This article is protected by copyright. All rights reserved. 
2. Time taken to improve in one grade on the GBS disability score

3. Time taken to walk independently

4. Difference in GBS disability score at maximum disability compared with 6 months

5. Percentage of participants with a clinically relevant improvement in R-ODS score defined as an increase from baseline in R-ODS score by at least 6 points on the centile metric score at 4 weeks and 6 months

6. Percentage of participants with a clinically relevant improvement in ONLS defined as a decrease from baseline in ONLS score by at least 1 point at 4 weeks and 6 months

7. Requirement for ventilatory support (GBS disability score $=5$ )

8. Duration of ventilatory support

9. Recurrence of relapse

10. Death within first 6 months

GBS DS, GBS Disability Score; RODS, Rasch Overall Disability Score; ONLS, Overall Neuropathy Limitation Score. Baseline defined as week 0, or day 1 prior to drug administration. 
Table 3. Baseline participant characteristics

\begin{tabular}{lccc} 
& $\begin{array}{c}\text { All } \\
(\mathbf{N = 8})\end{array}$ & $\begin{array}{c}\text { Eculizumab } \\
\mathbf{( N = 5 )}\end{array}$ & $\begin{array}{c}\text { Placebo } \\
\mathbf{( N = 2 )}\end{array}$ \\
\hline Gender & 4 & 2 & 2 \\
Male & 4 & 3 & 1 \\
Female & 4 & 3 & 1 \\
\hline Antecedent illness & 1 & 1 & 0 \\
None & 2 & 1 & 1 \\
Diarrhoea & 1 & 0 & 1 \\
Respiratory tract infection & & & \\
Urinary tract infection & $53(16)$ & $54(15)$ & $51(20)$ \\
\hline Age (years) & $32-72$ & $36-70$ & $32-72$ \\
Mean (SD) & & & \\
Range & 7 & 5 & 2 \\
\hline GBS disability score* & & & \\
Bed or chair bound (grade 4) & 4 & 2 & 2 \\
\hline Neurophysiology* & 2 & 2 & 0 \\
Demyelinating & 1 & 1 & 0 \\
Axonal & 5 & 4 & 1 \\
Equivocal & 3 & 3 & 0 \\
\hline Anti-ganglioside antibodies* & 2 & 1 & 1 \\
GM1 & & & \\
GM2 & &
\end{tabular}

Summary of baseline characteristics for gender, antecedent illness, age, disability score, clinical neurophysiology results as defined by local neurophysiology department and autoantibodies. Data shown are number of participants, unless otherwise stated.

* data for subject 8 who was withdrawn immediately after randomization and prior to treatment is not reported. 
Table 4 Primary and Secondary Outcomes

$\begin{array}{ccc}\text { All } & \begin{array}{c}\text { Eculizumab } \\ (\mathrm{N}=7)\end{array} & \begin{array}{c}\text { Placebo } \\ (\mathrm{N}=5)\end{array} \\ & & (\mathrm{N}=2)\end{array}$

\section{Primary outcome}

\section{Safety and tolerability}

Patients with adverse events

Total adverse events
Related to study drug

Total adverse events
Related to study drug

N

$7(100 \%)$

$5(100 \%)$

$2(100 \%)$

Yes

29

25

4

No

$9(31 \%)$

$9(36 \%)$

$0(0 \%)$

$20(69 \%)$

$16(64 \%)$

$4(100 \%)$

Patients with serious adverse

Total serious adverse events

Related to study drug

$\begin{array}{cccc} & 4(57 \%) & 4(80 \%) & 0(0 \%) \\ \text { N } & 4 & 4 & 0 \\ \text { Yes } & 1(25 \%) & 1(25 \%) & 0(0 \%) \\ \text { No } & 3(75 \%) & 3(75 \%) & 0(0 \%)\end{array}$

Death

$1(14 \%)$

$1(20 \%)$

$0(0 \%)$

\section{Efficacy}

GBS DS improvement from week

0 to week $4(\geq 1)$

Yes $\quad 4(57 \%) \quad 2(40 \%) \quad 2(100 \%)$

No

$3(43 \%)$

$3(60 \%)$

$0(0 \%)$

\section{Secondary Outcomes}

Independent walking at 8 weeks

$\begin{array}{lllc}\text { Yes } & 2(29 \%) & 1(20 \%) & 1(50 \%) \\ \text { No } & 5(71 \%) & 4(80 \%) & 1(50 \%) \\ \text { Yes } & 4(57 \%) & 2(40 \%) & 2(100 \%) \\ \text { No } & 3(43 \%) & 3(60 \%) & 0(0 \%)\end{array}$

Requirement for ventilation during trial

Yes $2(29 \%) \quad 2(40 \%) \quad 0(0 \%)$

No

$5(71 \%)$

$3(60 \%)$

$2(100 \%)$

Duration of ventilator support

Range

0-21

$1-21$

0

Primary efficacy outcomes described by groups. For individual case summaries, refer to appendix 2. Values given as absolute totals and percentages, unless otherwise stated.

This article is protected by copyright. All rights reserved. 
Independent walking classified as GBS DS $\leq 2$. Mechanical ventilation dependent classified as GBS DS 5

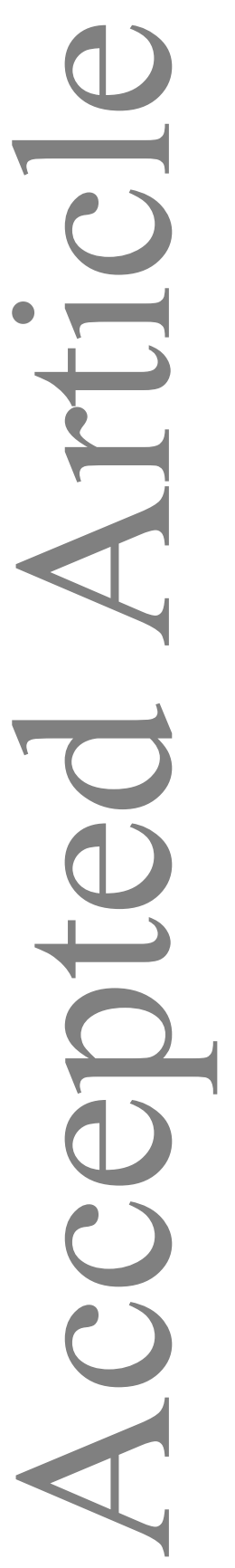

This article is protected by copyright. All rights reserved. 
Table 5. Observed versus predicted probability of walking

Probability of walking independently (\%; GBS DS $\leq 2$ ), based on mEGOS classifications at weeks 4,13 and 26 , versus observed walking status. Walking is defined as GBS DS 2 or less. E1-E5, eculizumab-treated subjects; P1-P2 placebotreated subjects.

\begin{tabular}{|c|c|c|c|c|c|c|c|c|c|c|c|c|c|c|}
\hline & \multicolumn{2}{|c|}{$\begin{array}{c}\mathrm{E1} \\
\mathrm{mEGOS}\end{array}=4$} & \multicolumn{2}{|c|}{$\begin{array}{c}\mathrm{E2} \\
\mathrm{mEGOS}=6\end{array}$} & \multicolumn{2}{|c|}{$\begin{array}{c}\mathrm{E3} \\
\mathrm{mEGOS}=11\end{array}$} & \multicolumn{2}{|c|}{$\begin{array}{c}\mathrm{E} 4 \\
\mathrm{mEGOS}\end{array}=7$} & \multicolumn{2}{|c|}{$\begin{array}{c}\mathrm{E5} \\
\mathrm{mEGOS}\end{array}$} & \multicolumn{2}{|c|}{$\begin{array}{c}\mathrm{P1} \\
\mathrm{mEGOS}=6\end{array}$} & \multicolumn{2}{|c|}{$\begin{array}{c}\mathrm{P} 2 \\
\mathrm{mEGOS}\end{array}=4$} \\
\hline & Pred & Obs & Pred & Obs & Pred & Obs & Pred & Obs & Pred & Obs & Pred & Obs & Pred & Obs \\
\hline 4 weeks & $60 \%$ & No & $40 \%$ & No & $2 \%$ & No & $30 \%$ & No & $50 \%$ & No & $40 \%$ & Yes & $60 \%$ & No \\
\hline $\begin{array}{l}13 \\
\text { weeks }\end{array}$ & $90 \%$ & Yes & $80 \%$ & Yes & $30 \%$ & No & $70 \%$ & No & $85 \%$ & No & $80 \%$ & Yes & $90 \%$ & Yes \\
\hline $\begin{array}{l}26 \\
\text { weeks }\end{array}$ & $95 \%$ & Yes & $90 \%$ & Yes & $50 \%$ & No & $85 \%$ & No & $93 \%$ & No & $90 \%$ & Yes & $95 \%$ & Yes \\
\hline
\end{tabular}

Pred: Predicted; Obs: Observed 


\section{References}

Asbury AK, Cornblath DR (1990). Assessment of current diagnostic criteria for Guillain-Barre syndrome. Ann Neurol 27 Suppl:S21-24.

Cao-Lormeau VM, Blake A, Mons S, Lastere S, Roche C, Vanhomwegen J, Dub T, Baudouin L, Teissier A, Larre P, Vial AL, Decam C, Choumet V, Halstead SK, Willison HJ, Musset L, Manuguerra JC, Despres P, Fournier E, Mallet HP, Musso D, Fontanet A, Neil J, Ghawche F (2016). Guillain-Barre Syndrome outbreak associated with Zika virus infection in French Polynesia: a case-control study. Lancet (London, England) 387:1531-1539.

Draak TH, Vanhoutte EK, van Nes SI, Gorson KC, Van der Pol WL, Notermans NC, Nobile-Orazio E, Leger JM, Van den Bergh PY, Lauria G, Bril V, Katzberg H, Lunn MP, Pouget J, van der Kooi AJ, Hahn AF, Doorn PA, Cornblath DR, van den Berg LH, Faber CG, Merkies IS (2014). Changing outcome in inflammatory neuropathies: Rasch-comparative responsiveness. Neurology 83:2124-2132.

Fitzpatrick AM, Mann CA, Barry S, Brennan K, Overell JR, Willison HJ (2011). An open label clinical trial of complement inhibition in multifocal motor neuropathy. J Peripher Nerv Syst 16:84-91.

Goodfellow JA, Willison HJ (2016). Antiganglioside, antiganglioside-complex, and antiglycolipidcomplex antibodies in immune-mediated neuropathies. Curr Opin Neurol 29:572-580.

Goodyear CS, O'Hanlon GM, Plomp JJ, Wagner ER, Morrison I, Veitch J, Cochrane L, Bullens RW, Molenaar PC, Conner J, Willison HJ (1999). Monoclonal antibodies raised against Guillain-Barre syndrome-associated Campylobacter jejuni lipopolysaccharides react with neuronal gangliosides and paralyze muscle-nerve preparations. J Clin Invest 104:697-708.

Graham RC, Hughes RA (2006). A modified peripheral neuropathy scale: the Overall Neuropathy Limitations Scale. Journal of neurology, neurosurgery, and psychiatry 77:973-976.

Greenshields KN, Halstead SK, Zitman FM, Rinaldi S, Brennan KM, O'Leary C, Chamberlain LH, Easton A, Roxburgh J, Pediani J, Furukawa K, Furukawa K, Goodyear CS, Plomp JJ, Willison HJ (2009). The neuropathic potential of anti-GM1 autoantibodies is regulated by the local glycolipid environment in mice. J Clin Invest 119:595-610.

Griffin JW, Li CY, Ho TW, Tian M, Gao CY, Xue P, Mishu B, Cornblath DR, Macko C, McKhann GM, Asbury AK (1996). Pathology of the motor-sensory axonal Guillain-Barre syndrome. Ann Neurol 39:17-28.

Halstead SK, Zitman FM, Humphreys PD, Greenshields K, Verschuuren JJ, Jacobs BC, Rother RP, Plomp JJ, Willison HJ (2008). Eculizumab prevents anti-ganglioside antibody-mediated neuropathy in a murine model. Brain 131:1197-1208.

Hughes RA, Newsom-Davis JM, Perkin GD, Pierce JM (1978). Controlled trial prednisolone in acute polyneuropathy. Lancet (London, England) 2:750-753.

Hughes RA, Pritchard J, Hadden RD (2013). Pharmacological treatment other than corticosteroids, intravenous immunoglobulin and plasma exchange for Guillain-Barre syndrome. Cochrane Database Syst Rev:Cd008630.

Hughes RA, Swan AV, van Doorn PA (2014). Intravenous immunoglobulin for Guillain-Barre syndrome. Cochrane Database Syst Rev:Cd002063.

This article is protected by copyright. All rights reserved. 
Kleyweg RP, van der Meche FG, Schmitz PI (1991). Interobserver agreement in the assessment of muscle strength and functional abilities in Guillain-Barre syndrome. Muscle \& nerve 14:11031109.

O'Hanlon GM, Humphreys PD, Goldman RS, Halstead SK, Bullens RW, Plomp JJ, Ushkaryov Y, Willison HJ (2003). Calpain inhibitors protect against axonal degeneration in a model of antiganglioside antibody-mediated motor nerve terminal injury. Brain 126:2497-2509.

Oomes PG, van der Meche FG, Kleyweg RP (1996). Liver function disturbances in Guillain-Barre syndrome: a prospective longitudinal study in 100 patients. Dutch Guillain-Barre Study Group. Neurology 46:96-100.

Rajabally YA, Durand MC, Mitchell J, Orlikowski D, Nicolas G (2015). Electrophysiological diagnosis of Guillain-Barre syndrome subtype: could a single study suffice? Journal of neurology, neurosurgery, and psychiatry 86:115-119.

Rother RP, Rollins SA, Mojcik CF, Brodsky RA, Bell L (2007). Discovery and development of the complement inhibitor eculizumab for the treatment of paroxysmal nocturnal hemoglobinuria. Nat Biotechnol 25:1256-1264.

van Nes SI, Vanhoutte EK, van Doorn PA, Hermans M, Bakkers M, Kuitwaard K, Faber CG, Merkies IS (2011). Rasch-built Overall Disability Scale (R-ODS) for immune-mediated peripheral neuropathies. Neurology 76:337-345.

van Schaik IN, Bossuyt PM, Brand A, Vermeulen M (1995). Diagnostic value of GM1 antibodies in motor neuron disorders and neuropathies: a meta-analysis. Neurology 45:1570-1577. Walgaard C, Lingsma HF, Ruts L, Drenthen J, van Koningsveld R, Garssen MJ, van Doorn PA, Steyerberg EW, Jacobs BC (2010). Prediction of respiratory insufficiency in Guillain-Barre syndrome. Ann Neurol 67:781-787.

Walgaard C, Lingsma HF, Ruts L, van Doorn PA, Steyerberg EW, Jacobs BC (2011). Early recognition of poor prognosis in Guillain-Barre syndrome. Neurology 76:968-975.

Willison HJ (2005). The immunobiology of Guillain-Barre syndromes. J Peripher Nerv Syst 10:94112.

Willison HJ, Veitch J, Swan AV, Baumann N, Comi G, Gregson NA, Illa I, Zielasek J, Hughes RA (1999). Inter-laboratory validation of an ELISA for the determination of serum anti-ganglioside antibodies. Eur J Neurol 6:71-77.

Yuki N, Yamada M, Koga M, Odaka M, Susuki K, Tagawa Y, Ueda S, Kasama T, Ohnishi A, Hayashi $\mathrm{S}$, Takahashi H, Kamijo M, Hirata K (2001). Animal model of axonal Guillain-Barre syndrome induced by sensitization with GM1 ganglioside. Ann Neurol 49:712-720.

This article is protected by copyright. All rights reserved. 


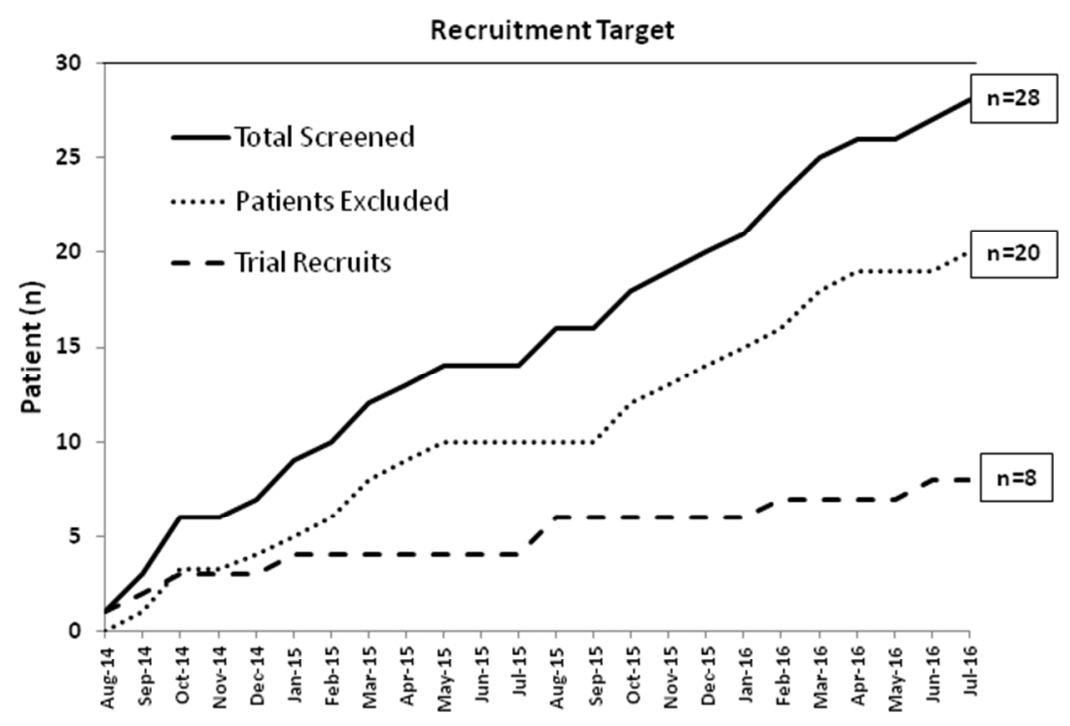

Figure 1: Recruitment log for screened subjects

Solid line denotes total GBS subjects at Hughes GBS DS grade 3 or greater undergoing screening for inclusion (28); dotted line denotes subjects excluded or who declined to participate (20); dashed line denotes subjects recruited into the study (8). The recruitment target was 30 subjects over 2 years. Of the 20 subjects who were excluded, 12 declined to participate and 8 were excluded for failing to meet other inclusion criteria. 
A

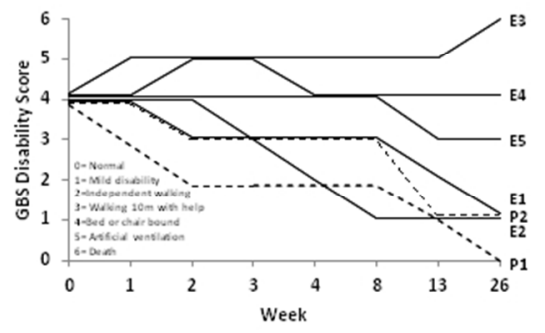

C

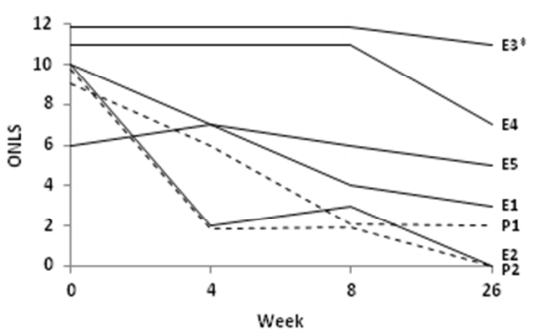

B

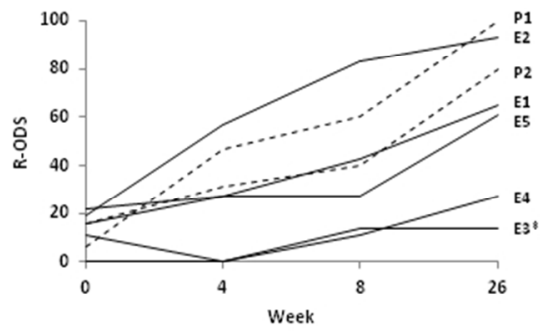

D

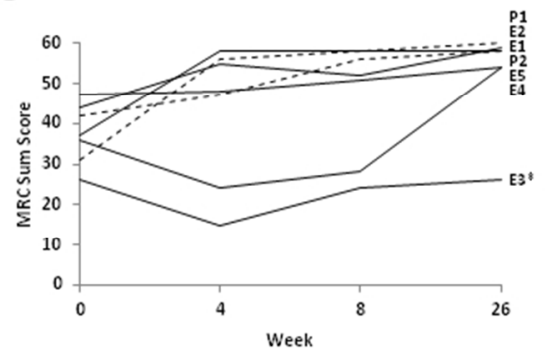

Figure 2: Efficacy Outcome markers plotted over time for ICA-GBS cohort

Efficacy outcome measurements in individual subjects over time. Panel A denotes trends in GBS disability scores at all trial time points (weeks 0-4, 8, 13, 26). Panel B denotes RODS (Rasch Overall Disability Score) outcomes (weeks 0, 4, 8, 26). Panel C denotes MRC sum score values per patient (weeks 0, 4, 8, 26). Panel D denotes ONLS (Overall Neuropathy Limitation Score) outcomes. Solid black line = eculizumabtreated Subjects, dashed black line = placebo-treated subjects. *In panels B-D endpoint data for E3 (who died at week 21) is week 13 data (not week 26). 